\title{
Staying safe in the community: adaptation of WaySafe to help probationers make better decisions about their health risks
}

\author{
Wayne EK Lehman*, Jennifer Pankow, Kevin Knight, Grace A Rowan, Julie Gray \\ From 2014 Addiction Health Services Research (AHSR) Conference \\ Boston, MA, USA. 15-17 October 2014
}

Transition from incarceration to the community is a critical time for offenders, especially those with substance abuse problems. Many succumb to temptation to return to drug use and possibly risky needle sharing, and engaging in risky sex activities. This critical time period entails substantial health risks for the offender as well as significant risks to public health.

Well-established and consistent use of $\mathrm{HIV} / \mathrm{HBV} / \mathrm{HCV}$ risk reduction prevention programs with continuity of care do not exist in most criminal justice treatment systems because of lack of policy development and integration between institution and community-based corrections, health, and social service agencies. Interventions targeting re-entry are crucial because of the likelihood for risk behaviors to increase upon return to the community. Approaches for community correction populations are needed that have the capability of addressing motivational, social, and cognitive deficits.

This presentation discusses an approach to translating ideas from the prison-based WaySafe curriculum into community settings for probationers who have recently completed residential, intensive outpatient, or prisonbased substance abuse treatment. The adapted intervention, StaySafe, will be based on evidence-based cognitive principles, including TCU Mapping Enhanced Counseling techniques designed to improve decision-making skills regarding health risk behaviors during the critical first 6 months under community supervision. It is being designed to be self-administered by participating probationers during downtime, either prior to or following meetings with their probation officers.

* Correspondence: w.lehman@tcu.edu

Institute of Behavioral Research, Texas Christian University, Fort Worth, TX, 76129, USA
An important goal in the adaptation and testing of StaySafe is to have a sustainable, evidence-based product for probation departments that can be administered with minimal staff training and time that is engaging and easy to use by probationers, requires minimal maintenance, and is free to probation departments (other than the cost of the touch-screen computers).

In this presentation, we describe the StaySafe intervention and the adaptation process from a prison-based group curriculum to a community corrections-based individualized approach. The adaptation process borrows ideas from the ADAPT-ITT framework [1]. We will describe initial results from focus groups and interviews with probationers and probation officers on problems and successes in probation, logistical issues with delivering interventions in probation settings, and results of a "theater" test of elements of StaySafe to gauge participant reactions.

\section{Acknowledgements \\ Abuse, National Institutes of Health (NIDA/NIH) through a grant to Texas Christian University (R01DA025885; Wayne E.K. Lehman, Principal Investigator). Interpretations and conclusions in this paper are entirely those}

Published: 20 February 2015

Reference

Wingood GM, DiClemente RJ: The ADAPT-ITT model: A novel method of adapting evidence-based HIV interventions. JAIDS 2008, 47:S40-S46.

doi:10.1186/1940-0640-10-S1-A32

Cite this article as: Lehman et al:: Staying safe in the community: adaptation of WaySafe to help probationers make better decisions about their health risks. Addiction Science \& Clinical Practice 2015 10(Suppl 1):A32. 